\title{
Mutual information, Fisher information and population coding
}

\author{
Nicolas Brunel and Jean-Pierre Nadal \\ Laboratoire de Physique Statistique de l'E.N.S. ${ }^{1}$ \\ Ecole Normale Supérieure \\ 24, rue Lhomond, 75231 Paris Cedex 05, France. \\ email: brunel@lps.ens.fr, nadal@lps.ens.fr.
}

\begin{abstract}
In the context of parameter estimation and model selection, it is only quite recently that a direct link between the Fisher information and information theoretic quantities has been exhibited. We give an interpretation of this link within the standard framework of information theory. We show that in the context of population coding, the mutual information between the activity of a large array of neurons and a stimulus to which the neurons are tuned is naturally related to the Fisher information. In the light of this result we consider the optimization of the tuning curves parameters in the case of neurons responding to a stimulus represented by an angular variable.
\end{abstract}

To appear in Neural Computation Vol. 10, Issue 7, published by the MIT press.

\footnotetext{
${ }^{1}$ Laboratory associated with C.N.R.S. (U.R.A. 1306), ENS, and Universities Paris VI and Paris VII
} 


\section{Introduction}

A natural framework to study how neurons communicate, or transmit information, in the nervous system is information theory (see e.g. Blahut 1988, Cover and Thomas 1991). In recent years the use of information theory in neuroscience has motivated a large number of works (e.g. Laughlin 1981, Linsker 1988, Barlow et al 1989, Bialek et al 1991, van Hateren 1992, Atick 1992, Nadal and Parga 1994). A neurophysiologist often asks in an informal sense how much information the spike train of a single neuron, or of a population of neurons, provides about an external stimulus. For example, a high activity of a CA3 hippocampal neuron may tell with good precision where a rat is in an environment. Information theory provides mathematical tools for measuring this 'information' or 'selectivity': signals are characterized by a probability distribution, and the spike train of a neuron, or of a population, is characterized by a probability distribution conditioned by the signal. The mutual information between the signal and the neural representation is then a measure of the statistical dependency between the signal and the spike train(s).

A related domain, which also belongs to information theory, is the field of statistical parameter estimation: here one typically has a sample of observations drawn from a distribution which depends on a parameter, or a set of parameters, that one wishes to estimate. The Cramer-Rao inequality then tells us that the mean squared error of any unbiased estimator of the underlying parameter(s) is lower bounded by the inverse of a quantity which is defined as the Fisher information (Blahut 1988). This means that the Fisher information is a measure of how well one can possibly estimate a parameter from an observation with a given probability law. Thus in this sense it is also an 'information' quantity.

In spite of the similar intuitive meanings of these two quantities, an explicit relationship between the Fisher information and information theoretic quantities has only been derived recently (Clarke and Barron 1990, Rissanen 1996), in the limit of a large number of observations. This link has been exhibited first in the context of parameter estimation (Clarke and Barron 1990) for the case of statistically independent and identically distributed observations. Then it has been generalized to a broader context within the framework of stochastic complexity, with as a result a refined "minimum description length" criterion for model selection (Rissanen 1996).

The first goal of this paper is to show that, within the framework of information theory, this link manifests itself very naturally in the context of neural coding: 
- In the limit of a large number of neurons coding for a low dimensional stimulus (population coding) the mutual information between the activities of the neuronal population and the stimulus becomes equal to the mutual information between the stimulus and an efficient Gaussian estimator, under appropriate conditions, detailed in Section 3. Here 'efficient' means that the variance of this estimator reaches the Cramer-Rao bound. Since this variance is related to the Fisher information, the abovementioned equality provides a quantitative link between mutual and Fisher informations.

- This equality is also shown to hold for a single cell in the case of a Gaussian noise with vanishing variance, in Section 4;

- The mutual information between the stimulus and an efficient Gaussian estimator reaches the mutual information between stimulus and the neuronal activities asymptotically from below.

In the light of this relationship between Fisher and mutual informations, we then examine in Section 5 several issues related to population codes, using neurons coding for an angular variable with a triangular or bell-shaped tuning curve. Such neurons are common in many neural structures. Cells of the postsubiculum (Taube et al 1990) and anterior thalamic nuclei (Taube 1995) of the rat are tuned to its head direction. Cells in MT cortex (Maunsell and Van Essen 1983) of the monkey are tuned to the direction of perceived motion. Cells in motor cortex of the monkey (Georgopoulos et al 1982) are tuned to the direction of the arm. We study the case of an array of $N$ neurons, firing as a Poisson process in response to an angular stimulus with a frequency defined by the tuning curve of the neuron, in an interval of duration $t$. In many cases Poisson processes are considered to be reasonable approximations of the firing process of cortical neurons (see e.g. Softky and Koch 1993).

We calculate the Fisher information with an arbitrary density of preferred angles. Next we address the question of the optimization of the tuning curves, making use of the link between mutual information and Fisher information. The optimal density of preferred angles (i.e. the one that maximizes the mutual information) is calculated as a function of the distribution of angles, in Section 5.2. As shown by Seung and Sompolinsky, the Fisher information, in the large $N$ limit, diverges when the tuning width of the neurons goes to zero. We show in Section 5.3 that a finite tuning width stems from optimization criteria which consider a finite system in which only a small 
number of spikes has been emitted by the whole population. We illustrate our results using triangular tuning curves in Section 5.4.

\section{General framework}

\subsection{Parameter estimation and population coding}

In the general context of "parameter estimation", one wishes to estimate a parameter $\theta$ from a set of $N$ observations $\left\{x_{i}, i=1, \ldots, N\right\} \equiv \vec{x}$ (where the $x_{i}$ 's might be discrete or continuous). $\theta$ may characterize a model $P(\vec{x} \mid \theta)$ which is expected to be a good description of the stochastic process generating the observations $\left\{x_{i}\right\}$. In the simplest case, the $x_{i}$ 's are independent realizations of the same random variable, and

$$
P(\vec{x} \mid \theta)=\prod_{i=1}^{N} p\left(x_{i} \mid \theta\right)
$$

It may be the case — but this is not necessary — that the true process $p^{*}(x)$ belongs to the family under consideration, so that $p^{*}(x)=p\left(x \mid \theta_{t}\right)$ where $\theta_{t}$ is the true value of the parameter.

In the context of sensory coding, and more specifically population coding (see e.g. Seung and Sompolinsky 1993, Snippe 1996) $\theta$ is a stimulus (e.g. an angle), and the information about this stimulus is contained in the activities $\left\{x_{i}, i=1, \ldots, N\right\}$ of a population of a large number $N$ of neurons. In the simplest case $x_{i}$ represents the activity of the $i$ th neuron of the output layer of a feedforward network with no lateral connection, so that the probability density function (p.d.f.) $P(\vec{x} \mid \theta)$ is factorized:

$$
P(\vec{x} \mid \theta)=\prod_{i=1}^{N} p_{i}\left(x_{i} \mid \theta\right) .
$$

Here $p_{i}\left(x_{i} \mid \theta\right)$ is the (neuron dependent) p.d.f. of the activity $x_{i}$ at neuron $i$ when the input stimulus takes the value $\theta$.

If the task of the neural system is to obtain a good estimate of the stimulus value, the problem is a particular case of parameter estimation where there does exist a true value - the one which generated the observed activity $\vec{x}$. 


\subsection{The Cramer-Rao bound}

In general one can find different algorithms for computing an estimate $\hat{\theta}(\vec{x})$ of $\theta$ from the observation of $\vec{x}$. If the chosen estimator $\hat{\theta}$ (algorithm) is unbiased, that is if

$$
\int d^{N} x P(\vec{x} \mid \theta) \hat{\theta}(\vec{x})=\theta
$$

the variance of the estimator

$$
\sigma_{\theta}^{2}=\left\langle(\hat{\theta}-\theta)^{2}\right\rangle_{\theta}
$$

in which $\langle\cdot\rangle_{\theta}$ denotes the integration over $\vec{x}$ given $\theta$ (a sum in the case of a discrete state vector) with the p.d.f. $P(\vec{x} \mid \theta)$, is bounded below according to (Cramer-Rao bound, see e.g. Blahut 1988):

$$
\sigma_{\theta}^{2} \geq \frac{1}{\mathcal{J}(\theta)}
$$

where $\mathcal{J}(\theta)$ is the Fisher information:

$$
\mathcal{J}(\theta)=\left\langle-\frac{\partial^{2} \ln P(\vec{x} \mid \theta)}{\partial \theta^{2}}\right\rangle_{\theta} .
$$

For a multidimensional parameter, Eq. (3) is replaced by an inequality for the covariance matrix, with $\mathcal{J}(\theta)$, the "Fisher information matrix", being then expressed in terms of the second derivatives of $\ln P(\vec{x} \mid \theta)$ (Blahut 1988). For simplicity we will restrict the discussion to the case of a scalar parameter, and consider the straightforward extension to the multidimensional case in section 3.2 .

An efficient estimator is an estimator which saturates the bound. The maximum likelihood (ML) estimator is known to be efficient in the large $N$ limit.

\section{Mutual information and Fisher information}

\subsection{Main result}

We now give the interpretation of the Cramer-Rao bound in terms of information content. First, one should note that the Fisher information, Eq. (4) is not, itself, an information quantity. The terminology comes from an intuitive interpretation of the bound: our knowledge ("information") about a stimulus $\theta$ is limited according to this bound. This qualitative statement has been turned into a quantitative statement in (Clarke and Barron 1990, Rissanen 1996). We give here a different presentation based 
on a standard information theoretic point of view, which is relevant for sensory coding, rather than from the point of view of parameter estimation and model selection.

We consider the mutual information between the observable $\vec{x}$ and the stimulus $\theta$. It can be defined very naturally in the context of sensory coding because $\theta$ is itself a random quantity, generated with some p.d.f. $\rho(\theta)$ which characterizes the environment. The mutual information is defined by (Blahut 1988):

$$
I[\theta, \vec{x}]=\int d \theta d^{N} x \rho(\theta) P(\vec{x} \mid \theta) \log \frac{P(\vec{x} \mid \theta)}{Q(\vec{x})}
$$

where $Q(\vec{x})$ is the p.d.f. of $\vec{x}$ :

$$
Q(\vec{x})=\int d \theta \rho(\theta) P(\vec{x} \mid \theta)
$$

Other measures of the statistical dependency between input and output could be considered, but the mutual information is the only one (up to a multiplicative constant) satisfying a set of fundamental requirements (Shannon and Weaver 1949).

Suppose there exists an unbiased efficient estimator $\hat{\theta}=T(\vec{x})$. It has mean $\theta$ and variance $1 / \mathcal{J}(\theta)$. The amount of information gained about $\theta$ in the computation of that estimator is

$$
I[\theta, \hat{\theta}]=\mathcal{H}[\hat{\theta}]-\int d \theta \rho(\theta) \mathcal{H}[\hat{\theta} \mid \theta]
$$

where $\mathcal{H}[\hat{\theta}]$ is the entropy of the estimator,

$$
\mathcal{H}[\hat{\theta}]=-\int d \hat{\theta} \operatorname{Pr}(\hat{\theta}) \ln \operatorname{Pr}(\hat{\theta})
$$

and $\mathcal{H}[\hat{\theta} \mid \theta]$ its entropy given $\theta$. The latter, for each $\theta$, is smaller than the entropy of a Gaussian distribution with the same variance $1 / \mathcal{J}(\theta)$. This implies

$$
I[\theta, \hat{\theta}] \geq \mathcal{H}[\hat{\theta}]-\int d \theta \rho(\theta) \frac{1}{2} \ln \left(\frac{2 \pi e}{\mathcal{J}(\theta)}\right)
$$

Since processing cannot increase information (see e.g. Blahut 1988, pp. 158-159), the information $I[\theta, \vec{x}]$ conveyed by $\vec{x}$ about $\theta$ is at least equal to the one conveyed by the estimator: $I[\theta, \vec{x}] \geq I[\theta, \hat{\theta}]$. For the efficient estimator, this means

$$
I[\theta, \vec{x}] \geq \mathcal{H}[\hat{\theta}]-\int d \theta \rho(\theta) \frac{1}{2} \ln \left(\frac{2 \pi e}{\mathcal{J}(\theta)}\right)
$$

In the limit in which the distribution of the estimator is sharply peaked around its mean value, (in particular this implies $\mathcal{J}(\theta) \gg 1$ ), the entropy of the estimator 
becomes identical to the entropy of the stimulus. The r.h.s in the above inequality becomes then equal to $I_{\text {Fisher }}$ plus terms of order $1 / \mathcal{J}(\theta)$, with $I_{\text {Fisher }}$ defined as

$$
I_{\text {Fisher }}=\mathcal{H}(\Theta)-\int d \theta \rho(\theta) \frac{1}{2} \ln \left(\frac{2 \pi e}{\mathcal{J}(\theta)}\right)
$$

In the above expression the first term is the entropy of the stimulus,

$$
\mathcal{H}(\theta)=-\int d \theta \rho(\theta) \ln \rho(\theta)
$$

For a discrete distribution this would be the information gain resulting from a perfect knowledge of $\theta$. The second term is the equivocation due to the Gaussian fluctuations of the estimator around its mean value. We thus have, in this limit of a good estimator,

$$
I[\theta, \vec{x}] \geq I_{\text {Fisher }}
$$

The inequality (12), with $I_{F i s h e r}$ given by (10), gives the essence of the link between mutual information and Fisher information. It results from an elementary application of the simple - but fundamental - theorem on information processing, and of the Cramer-Rao bound.

Now if the Cramer-Rao bound was to be understood as a statement on information content, $I[\theta, \vec{x}]$ could not be strictly larger than $I_{\text {Fisher }}$ - if not there would be a way to extract from $\vec{x}$ more information than $I_{F i s h e r}$. Hence the above inequality would be in fact an equality, that is:

$$
I[\theta, \vec{x}]=-\int d \theta \rho(\theta) \ln \rho(\theta)-\int d \theta \rho(\theta) \frac{1}{2} \ln \left(\frac{2 \pi e}{\mathcal{J}(\theta)}\right)
$$

However, the fact that the equality should hold is not obvious: the Cramer-Rao bound does not tell us whether knowledge on other cumulants than the variance could be obtained. Indeed, if the estimator has a non Gaussian distribution, the inequality will be strict, and we will give an example in section 4 where we discuss the case of a single output cell $(N=1)$. In the large $N$ limit, however, there exists an efficient estimator (the maximum likelihood), and relevant probability distributions become close to Gaussian distributions, so that one can expect (13) to be true in that limit. This is indeed the case, and what is proved in (Rissanen 1996) within the framework of Stochastic Complexity, under suitable but not very restrictive hypotheses.

In Appendix, we show, using completely different techniques, that Eq. (13) holds provided the following conditions are satisfied: 
1. All derivatives of $G(\vec{x} \mid \theta) \equiv \ln P(\vec{x} \mid \theta) / N$ with respect to the stimulus $\theta$ are of order one;

2. The cumulants (with respect to the distribution $P(\vec{x} \mid \theta)$ ) of order $n$ of $a G_{\theta}^{\prime}+b G_{\theta}^{\prime \prime}$ are of order $1 / N^{n-1}$ for all $a, b, n$.

The meaning of the last condition is that, at a given value of $N$, the cumulants should decrease sufficiently rapidly with $n$. This is in particular true when $x_{i}$ given $\theta$ are independent, as for model (2), but holds also in the more general case when the $x_{i}$ are correlated, provided the above conditions hold, as we show explicitly in Appendix using an example of correlated $x_{i}$.

\subsection{Extensions and remarks}

\section{Multi-parameter case and model selection}

It is straightforward to extend Eq. (12) to the case of a $K$ dimensional stimulus $\vec{\theta}$ with p.d.f. $\rho(\vec{\theta})$, and to derive the equality Eq. (13) for $K \ll N$. The Fisher information matrix is defined as (Blahut 1988)

$$
\mathcal{J}_{i j}(\vec{\theta})=\left\langle-\frac{\partial^{2} \ln P(\vec{x} \mid \vec{\theta})}{\partial \theta_{i} \partial \theta_{j}}\right\rangle_{\vec{\theta}}
$$

The quantity $I_{F i s h e r}$ for the multidimensional case is then

$$
I_{\text {Fisher }}=-\int d^{K} \theta \rho(\vec{\theta}) \ln \rho(\vec{\theta})-\int d^{K} \theta \rho(\vec{\theta}) \frac{1}{2} \ln \left(\frac{(2 \pi e)^{K}}{\operatorname{det} \mathcal{J}(\vec{\theta})}\right)
$$

The second term is now equal to the entropy of a Gaussian with covariance matrix $\mathcal{J}^{-1}(\vec{\theta})$, averaged over $\vec{\theta}$ with p.d.f. $\rho(\vec{\theta})$. In the large $N$ limit $(K<<N)$, one gets as for $K=1$ the equality $I=I_{\text {Fisher }}$.

One can note that formulae (13) and (14) are also meaningful in the more general context of parameter estimation, even when $\theta$ is not a priori a random variable: within the Bayesian framework (Clarke and Barron, 1990), it is natural to introduce a prior distribution on the parameter space, $\rho(\theta)$. Typically, this distribution is chosen as the flattest possible one which takes into account any prior knowledge or constraint on the parameter space. Then $I$ tells us how well $\theta$ can be localized within the parameter space from the observation of the data $\vec{x}$.

Within the framework of MDL (minimum description length) (Rissanen 1996) the natural prior is the one which maximizes the mutual information, i.e. the one realizing 
the Shannon capacity. Maximizing $I=I_{\text {Fisher }}$ with respect to $\rho$, one finds that this optimal input distribution is given by the square root of the Fisher information:

$$
\rho(\theta)=\frac{\sqrt{\mathcal{J}(\theta)}}{\int d \theta^{\prime} \sqrt{\mathcal{J}\left(\theta^{\prime}\right)}}
$$

(for the multidimensional case, $\mathcal{J}$ in the above expression has to be replaced by $\operatorname{det} \mathcal{J}$ ). This corresponds to the stimulus distribution for which the neural system is best adapted.

\section{Biased estimators}

The preceeding discussions can be easily extended to the case of biased estimators, that is for estimators $\hat{\theta}$ with $<\hat{\theta}>_{\theta}=m(\theta) \neq \theta$. The Cramer-Rao bound in such a case reads

$$
\frac{\sigma_{\theta}^{2}}{\left(\frac{d m}{d \theta}\right)^{2}} \geq \frac{1}{\mathcal{J}(\theta)}
$$

This is a form of the bias-variance compromise. One can thus write an inequality similar to Eq. (8), replacing $\mathcal{J}$ by $\mathcal{J} /(d m / d \theta)^{2}$. In the limit where the estimator is sharply peaked around its mean value $m(\theta)$, one has $\rho(\theta) d \theta \sim P(\hat{\theta}) d \hat{\theta}$, and $\hat{\theta} \sim m(\theta)$, so that

$$
\mathcal{H}[\hat{\theta}]=\mathcal{H}[\theta]+\int d \theta \rho(\theta) \log \left|\frac{d m}{d \theta}\right|
$$

Upon inserting $\mathcal{H}[\hat{\theta}]$ in the r.h.s of the inequality (8), the terms $\frac{d m}{d \theta}$ cancel. The bound, Eq. (12), is thus also valid even when the known efficient estimator is biased.

The Cramer-Rao bound can also be understood as a bound for the discriminability $d^{\prime}$ used in psychophysics for characterizing performance in a discrimination task between $\theta$ and $\theta+\delta \theta$ (see e.g. Green and Swets, 1966). As discussed in (Seung and Sompolinsky, 1993)

$$
d^{\prime} \leq \delta \theta \sqrt{\mathcal{J}(\theta)}
$$

with equality for an efficient estimator, and with $d^{\prime}$ properly normalized with respect to the bias:

$$
d^{2}=\frac{\left(\delta \theta \frac{d m}{d \theta}\right)^{2}}{\sigma_{\theta}^{2}} .
$$




\section{The case of a single neuron}

\subsection{A continuous neuron with vanishing output noise}

We consider the case of a single neuron characterized by a scalar output $V$ which a deterministic function of the input (stimulus) $\theta$ plus some noise, with a possibly stimulus dependent variance:

$$
V=f(\theta)+z \sigma \sqrt{g(\theta)}
$$

where $f$ and $g$ are deterministic functions, and $\sigma$ is a parameter giving the scale of the variance of the noise, and $z$ is a random variable with an arbitrary (that is not necessarily Gaussian) distribution $Q(z)$ with zero mean and unit variance. We are interested in the low noise limit, $\sigma \rightarrow 0$. It is not difficult to write the Fisher information $\mathcal{J}(\theta)$ and the mutual information $I[\theta, V]$ in the limit of vanishing $\sigma$. One gets, for sufficiently regular $Q($.$) ,$

$$
I[\theta, V]=\mathcal{H}(\Theta)+\int d \theta \rho(\theta) \frac{1}{2} \log \frac{f^{\prime 2}(\theta)}{\sigma^{2} g(\theta)}-\mathcal{H}(Z)
$$

where $\mathcal{H}(Z)$ is the entropy of the $z$-distribution $Q$ :

$$
\mathcal{H}(Z)=-\int d z Q(z) \log Q(z)
$$

For the Fisher information one finds

$$
\mathcal{J}(\theta)=\frac{f^{\prime 2}(\theta)}{\sigma^{2} g(\theta)} \int d z \frac{Q^{\prime 2}(z)}{Q(z)}
$$

so that

$$
I_{\text {Fisher }}[\theta, V]=\mathcal{H}(\Theta)+\int d \theta \rho(\theta) \frac{1}{2} \log \frac{f^{\prime 2}(\theta)}{\sigma^{2} g(\theta)}+\frac{1}{2} \log \int d z \frac{Q^{\prime 2}(z)}{Q(z)}
$$

If the noise distribution $Q$ is the normal distribution, one has $\mathcal{H}(Z)=\frac{1}{2} \log 2 \pi e$, and the integral in Eq. (21) is equal to 1 , so that one has $I=I_{F i s h e r}$. Otherwise one can easily check that $I>I_{\text {Fisher }}$, in agreement with the general result (12).

\subsection{Optimization of the transfer function}

The maximization of the mutual information with respect to the choice of the transfer function $f$ has been studied in the case of a stimulus independent additive noise, that 
is $g \equiv 1$, by Laughlin (1981) and Nadal and Parga (1994). The expression (19) for the mutual information, with $g=1$, has been computed by Nadal and Parga (1994). What is new here is the link with the Fisher information.

The mutual information is maximized when $f$ is chosen according to the "equalization rule", that is when the (absolute value of) the derivative of $f$ is equal to the p.d.f. $\rho$ : the activity $V$ is then uniformly distributed between its min and max values. In the more general case in which $g$ depends on the stimulus, the maximum of $I$ is reached when $\hat{f}$ defined by

$$
\hat{f}^{\prime} \equiv f^{\prime} / \sqrt{g}
$$

satisfies the equalization rule

$$
\hat{f}=A \int^{\theta} d x \rho(x)+B
$$

where $A$ and $B$ are arbitrary given parameters (for $g=1$, they define the min and max values of $f$ ). An interesting case is $g=f$, which is relevant for the analysis of a Poisson neuron in the large time limit (see next subsection). In this case $f^{\prime} / \sqrt{g}=2 \sqrt{f}$, and the maximum of $I$ is reached when the square root of $f$ satisfies the equalization rule.

The fact that the mutual information is related to the Fisher information in the case of a single neuron with vanishing noise means that maximizing information transfer is identical to minimizing the variance of reconstruction error. In fact, two different qualitative lines of reasoning were known to lead to the equalization rule: one related to information transfer (the output $V$ should have a uniform distribution, see e.g. Laughlin 1981), and one related to reconstruction error (the slope of the transfer function should be as large as possible in order to minimize this error, and this, with the constraint that $f$ is bounded, leads to the compromise $\left|f^{\prime}\right|=\rho$ - a large error can be tolerated for rare events). We have shown here the formal link between these two approaches, using the link between mutual and Fisher informations.

\subsection{A Poisson neuron}

Another but related interesting case is the one of a single neuron emitting spikes according to a Poisson process (in the next section we will consider a population of such neurons). The probability for observing $k$ spikes in the interval $[0, t]$ while the stimulus $\theta$ is perceived, is

$$
p(k \mid \theta)=\frac{(\nu(\theta) t)^{k}}{k !} \exp (-\nu(\theta) t)
$$


where the frequency $\nu$ is assumed to be a deterministic function $\nu(\theta)$ (the tuning curve) of the stimulus $\theta$ :

$$
\theta \rightarrow \nu=\nu(\theta)
$$

If the stimulus is drawn randomly from a distribution $\rho(\theta)$, the frequency distribution $\mathcal{P}(\nu)$ is given by

$$
\mathcal{P}(\nu)=\int d \theta \rho(\theta) \delta(\nu-\nu(\theta))
$$

The information processing ability of such model neuron has been studied in great details by Stein (1967). The results of interest here are as follows.

At short times (that is for $t \mu$ small), the mutual information between the stimulus and the cell activity is, at first order in $t$ (Stein 1967)

$$
I(t) \sim t \int d \nu \mathcal{P}(\nu) \nu \log \frac{\nu}{\mu} \equiv I_{1}(t)
$$

where $\mu$ is the mean frequency. One can easily check that $I_{1}(t) \geq I(t)$ for any duration $t$. In fact at long times ( $t \mu$ large) information increases only as log $t$ : in the large time limit, one gets (Stein 1967)

$$
I(t)=\int d \nu \mathcal{P}(\nu) \log \left(\mathcal{P}(\nu) \sqrt{\frac{2 \pi e \nu}{t}}\right)
$$

From this expression, one gets that the optimal tuning curve is such that $\sqrt{\nu}$ is uniformly distributed between its extreme values $\nu_{\min }$ and $\nu_{\max }$. We can now analyze this result in view of the relationship between Fisher and mutual information. Making the change of variable $\nu \rightarrow \theta$, with

$$
\rho(\theta) d \theta=\mathcal{P}(\nu) d \nu
$$

together with Eq (25), one can rewrite the mutual information at large times precisely as

$$
I(t)=I_{\text {Fisher }}
$$

where $I_{\text {Fisher }}$ is defined as in $(10)$ whith $\mathcal{J}(\theta)$ the Fisher information associated to this single neuron:

$$
\mathcal{J}(\theta)=t \frac{\nu^{\prime 2}(\theta)}{\nu(\theta)}
$$

This result can be understood in the following way. In the limit of large $t$, the distribution of the number of emitted spikes divided by $t, V \equiv k / t$ tends to be a 
Gaussian, with mean $\nu(\theta)$ and variance $\nu(\theta) / t$, so that the properties of the spiking neuron become similar to those of a neuron having a continuous activity $V$, given by

$$
\theta \rightarrow V=\nu(\theta)+z \sqrt{\nu(\theta) / t}
$$

where $z$ is a Gaussian random variable with zero mean and unit variance. This is a particular case of Eq. (18) with $\sigma=1 / \sqrt{t}, f()=.g()=.\nu($.$) .$

\section{$5 \quad$ Population of direction selective spiking neurons}

\subsection{Fisher information}

We now illustrate the main statement of section 3 in the context of population coding. We consider a large number $N$ of neurons coding for a scalar stimulus, e.g. an angle. Eq. (13) tells us that to compute the mutual information we have first to calculate the Fisher information.

When the activities $\left\{x_{i}\right\}$ of the neurons given $\theta$ are independent, $P(\vec{x} \mid \theta)=\Pi p_{i}\left(x_{i} \mid \theta\right)$, the Fisher information can be written

$$
\mathcal{J}(\theta)=\sum_{i=1}^{N}\left\langle\frac{1}{p_{i}^{2}\left(x_{i} \mid \theta\right)}\left(\frac{\partial p_{i}\left(x_{i} \mid \theta\right)}{\partial \theta}\right)^{2}\right\rangle_{i, \theta}
$$

where $\langle.\rangle_{i, \theta}$ is the integration over $x_{i}$ with the p.d.f. $p_{i}\left(x_{i} \mid \theta\right)$.

We restrict ourselves to the case of neurons firing as a Poisson process with rate $\nu_{i}(\theta)$ in response to a stimulus $\theta \in[-\pi, \pi] . \nu_{i}(\theta)$ therefore represent the 'tuning curve' of neuron $i$. We make the following assumptions: $\nu_{i}(\theta)$ has a single maximum at the 'preferred stimulus' $\theta_{i}$; the tuning curve depends only on the distance between the current stimulus and the preferred one and is a periodic function of this distance

$$
\nu_{i}(\theta)=\phi\left(\theta-\theta_{i}\right)
$$

through the same function $\phi$. The locations of the preferred stimuli of the neurons are i.i.d. variables in the interval $\theta \in[-\pi, \pi]$ with density $r(\theta)$.

Since our model neurons fire as a Poisson process, the information contained in their spike trains in an interval of duration $t$ is fully contained in the number of spikes $x_{i}$ emitted by each neuron in this interval. For a Poisson process we have the law

$$
p_{i}\left(x_{i} \mid \theta\right)=\frac{\left(\nu_{i}(\theta) t\right)^{x_{i}}}{x_{i} !} \exp \left(-\nu_{i}(\theta) t\right)
$$




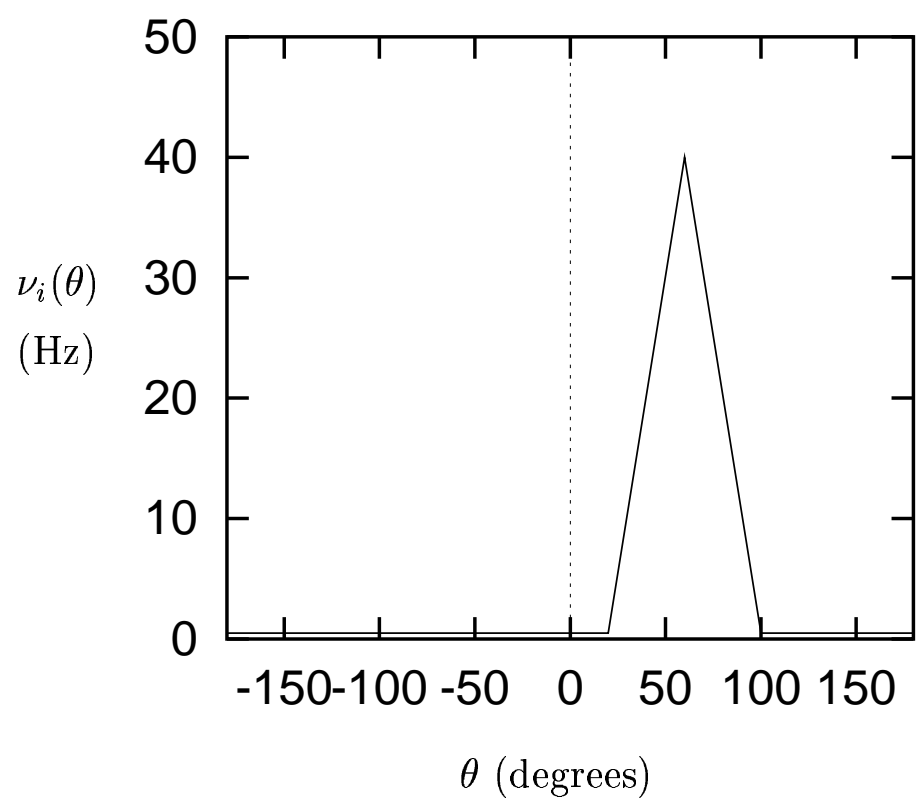

Figure 1: Triangular tuning curve corresponding to a minimal frequency $\nu_{\min }=0.5 \mathrm{~Hz}$, $\nu_{\text {max }}=40 \mathrm{~Hz}$, a receptive field half-width $a=40$ degrees, a preferred angle $\theta_{i}=60$ degrees.

From Eqs. $(31,33)$ we can easily calculate the Fisher information:

$$
\mathcal{J}(\theta)=t \sum_{i=1}^{N} \frac{\nu_{i}^{\prime}(\theta)^{2}}{\nu_{i}(\theta)}
$$

For $N$ large we can replace the sum by the average over the distribution of preferred stimuli, that is

$$
\overline{\mathcal{J}(\theta)}=t N \int_{-\pi}^{\pi} d \theta^{\prime} r\left(\theta^{\prime}\right) \frac{\phi^{\prime}\left(\theta-\theta^{\prime}\right)^{2}}{\phi\left(\theta-\theta^{\prime}\right)}
$$

For an isotropic distribution $r(\theta)=1 /(2 \pi)$ we recover the result of Seung and Sompolinsky (1993).

To understand how the Fisher information depends on other parameters of the tuning curve $\phi$ we redefine

$$
\phi\left(\theta-\theta_{i}\right)=\nu_{\min }+\left(\nu_{\max }-\nu_{\min }\right) \Phi\left(\frac{\left|\theta-\theta_{i}\right|}{a}\right)
$$

where $\nu_{\min }$ and $\nu_{\max }$ are the minimal and maximal frequency, $a$ is the width of the tuning curve, and $\Phi$ is a decreasing function of $\left|\theta-\theta_{i}\right| / a$ such that $\Phi=1$ for the preferred stimulus $\theta=\theta_{i}$, and $\Phi=0$ for stimuli far from the preferred stimulus, 
$\left|\theta-\theta_{i}\right| \gg a$. In terms of these parameters we have

$$
\overline{\mathcal{J}(\theta)}=t N \frac{\left(\nu_{\max }-\nu_{\min }\right)}{a} \int d z r(\theta+a z) \frac{\Phi^{\prime}(z)^{2}}{\frac{\nu_{\min }}{\nu_{\max }-\nu_{\min }}+\Phi(z)} .
$$

The particular case of a triangular tuning curve,

$$
\Phi(x)= \begin{cases}(1-|x|) & x \in[-1,1] \\ 0 . & |x|>1\end{cases}
$$

is shown in Fig. 1. It will be considered in more details below. For this tuning curve, and for a uniform distribution of preferred stimuli, the Fisher information has the simple form

$$
\overline{\mathcal{J}(\theta)}=t N \frac{\left(\nu_{\max }-\nu_{\min }\right)}{\pi a} \ln \frac{\nu_{\max }}{\nu_{\min }} .
$$

Thus, as already noted by (Seung and Sompolinsky 1993), the Fisher information diverges in different extreme cases: when the maximal frequency $\nu_{\max }$ goes to infinity; when the tuning width $a$ goes to zero. Moreover, functions $\Phi$ can be found such that

the Fisher information diverges (e.g. $\Phi(x)=\sqrt{1-x^{2}}$ ) for any value of $\nu_{\min }, \nu_{\max }$, and $a$. Thus the optimization of the Fisher information with respect to these parameters is an ill-defined problem without additional constraints. Note that in these cases the equation relating the Fisher information to the mutual information is no longer valid.

There is however a well-defined optimization problem which is the optimization with respect to the distribution of preferred orientations. It is considered in Section 5.2. Then we show how finite size effects transforms the problem of the optimization of both Fisher and mutual informations with respect to the tuning width $a$ into a well-defined problem, in Section 5.3. Last we present some numerical estimates of these quantities inserting some real data (Taube et al 1990) in Eq. (13), in Section 5.4 .

\subsection{Optimization over the distribution of preferred orienta- tions}

We ask the question of which distribution of preferred orientations $r(\theta)$ optimizes the mutual information $I$. Obviously the optimal $r$ will depend on the distribution of orientations $\rho(\theta)$. Optimizing Eq. (13) with respect to $r\left(\theta^{\prime}\right)$ subject to the normalization constraint $\int r\left(\theta^{\prime}\right) d \theta^{\prime}=1$ gives

$$
\int d \theta \frac{\rho(\theta)}{\int d \theta^{\prime \prime} r\left(\theta^{\prime \prime}\right) \psi\left(\theta-\theta^{\prime \prime}\right)} \psi\left(\theta-\theta^{\prime}\right)=c t \text { for all } \theta^{\prime}
$$


in which we have defined

$$
\psi(x)=\frac{\phi^{\prime}(x)^{2}}{\phi(x)}
$$

This condition is satisfied when

$$
\rho(\theta)=\frac{\int d \theta^{\prime} r\left(\theta^{\prime}\right) \psi\left(\theta-\theta^{\prime}\right)}{\int d \theta^{\prime} \psi\left(\theta^{\prime}\right)}
$$

Thus the optimal distribution of preferred stimuli is the one that, convolved with $\psi$ (i.e. a quantity proportional to the Fisher information) matches the distribution of stimuli. Of course in the particular case of $\rho(\theta)=1 /(2 \pi)$ we obtain $r_{\text {opt }}(\theta)=1 /(2 \pi)$. Note that Eq. (37) is also valid for unbounded stimulus values.

One should note that this result, Eq. (37) is specific to the optimization of the mutual information. Different results would be obtained for, e.g., the maximization of the average of the Fisher information, or the minimization of the average of its inverse. In fact, there is no optimum for the mean Fisher information, since it is linear in $r($.$) .$

\subsection{Finite size effects: the case of a single spike}

We have seen that the Fisher information, in the large $N$ limit, diverges when the tuning width $a$ goes to zero. To investigate whether this property is specific to the large $N$ limit we study the case of a finite number of neurons, in a very short time interval in which a single spike has been emitted by the whole population in response to the stimulus $\theta$. In this situation, it is clear that the optimal estimator of the stimulus (the ML estimate in that case) is given by the preferred stimulus of the neuron which emitted the spike. For finite $N$ the Cramer-Rao bound is in general not saturated, and we have to calculate directly the performance of the estimator. It is a simple exercise to calculate the standard deviation (SD) of the error made by such an estimate for a triangular tuning curve given in Eq. (34):

$$
\mathrm{SD}(\text { error })=\sqrt{\frac{4 \pi^{3} \nu_{\min }+a^{3}\left(\nu_{\max }-\nu_{\min }\right)}{6\left(2 \pi \nu_{\min }+a\left(\nu_{\max }-\nu_{\min }\right)\right)}}
$$

which always has a minimum for $0<a<\pi$. We show in Fig. 2 the SD of the reconstruction error after a single spike as a function of $a$, for $\nu_{\max } / \nu_{\min }=80$.

It has a minimum for $a$ about 50 degrees, for which the SD of the error is about 35 degrees. 

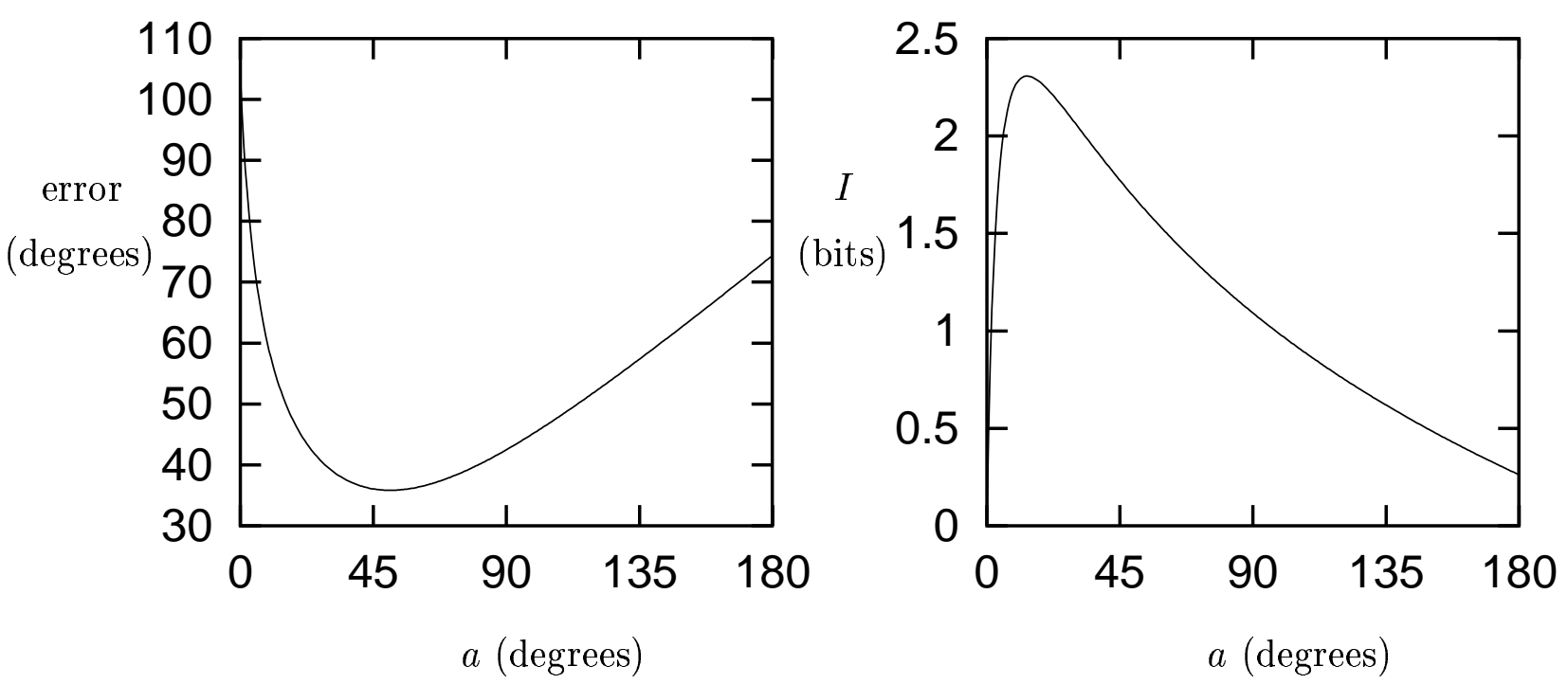

Figure 2: Left: SD of the reconstruction error after a single spike, as a function of $a$. Right: mutual information between the spike and the stimulus as a function of $a$. Note that minimizing the SD of the reconstruction error is in this case different than maximizing the mutual information.

The mutual information, on the other hand, is

$$
\begin{gathered}
I=\frac{1}{\pi \bar{\nu}}\left[\frac{a}{\nu_{\max }-\nu_{\min }}\left(\frac{\nu_{\max }^{2}}{2} \log \left(\frac{\nu_{\max }}{\bar{\nu}}\right)-\frac{\nu_{\min }^{2}}{2} \log \left(\frac{\nu_{\min }}{\bar{\nu}}\right)-\frac{1}{4}\left(\nu_{\max }^{2}-\nu_{\min }^{2}\right)\right)+\right. \\
\left.+(\pi-a) \nu_{\min } \log \left(\frac{\nu_{\min }}{\bar{\nu}}\right)\right]
\end{gathered}
$$

where

$$
\bar{\nu}=\nu_{\min }+\frac{a}{2 \pi}\left(\nu_{\max }-\nu_{\min }\right)
$$

It also has a maximum for positive $a$. The width that maximizes $I$ is different than the width that minimizes the SD of the reconstruction error, as shown in Fig. 2. This is the case in general for non-Gaussian tuning curves. In this case, the half-width maximizing the mutual information is around 20 degrees. Note that in a wide range of $a$ the first spike brings about 2 bits of information about the stimulus.

Thus a finite optimal $a$ stems from the constraint of already minimizing the error when only a small number of spikes have been emitted by the whole neuronal array. It implies that the largest receptive fields are most useful at very short times when only a rough estimate is possible, while smaller receptive fields will be most useful at larger times, when a more accurate estimate can be obtained. 


\subsection{Application to the analysis of empirical data}

In this section we use the experimental data of (Taube et al 1990) to show how Eq. (13) can be used to estimate both Fisher and mutual informations conveyed by large populations of neurons on an angular stimulus (in this case the head direction of a rat). Taube et al (1990) have shown that in the postsubiculum of rats tuning curves can be well fitted by triangular tuning curves, and that the distribution of preferred orientations is consistent with a uniform distribution. They also determined the distribution of the parameters of the tuning curve, $\nu_{\max }, a$ and the signal-tonoise ratio (SNR) $\alpha=\nu_{\max } / \nu_{\min }$ over the recorded neurons. This data indicate these parameters have an important variability from neuron to neuron. Eq. (35), in the case of such inhomogeneities, has to be replaced by

$$
\overline{\mathcal{J}(\theta)}=\frac{t N}{\pi} \int d \nu_{\max } d a d \alpha \operatorname{Pr}\left(\nu_{\max }, a, \alpha\right) \frac{\nu_{\max }}{a}\left(1-\frac{1}{\alpha}\right) \ln \alpha
$$

in which $\operatorname{Pr}\left(\nu_{\text {max }}, a, \alpha\right)$ is the joint probability of parameters $\nu_{\max }, a$ and $\alpha$. Under global constraints, one may expect each neuron to contribute in the same way to the information, that is $\left(\nu_{\max } / a\right)(1-1 / \alpha) \ln \alpha$ is constant. This would imply that the width $a$ increases with $\nu_{\max }$. Fig. 9 of (Taube et al 1990) show that there is indeed a trend for higher firing rate cells to have wider directional firing ranges.

We can now insert the distributions of parameters measured in Taube et al (1990) in Eq. (38) to estimate the minimal reconstruction error that can be done on the head direction using the output of $N$ postsubiculum neurons during an interval of duration $t$. It is shown in the left part of Fig. 3. Since we assume that the number of neurons is large, the mutual information conveyed by this population can be estimated using Eq. (13). It is shown in the right part of the same figure. In the case of $N=$ 5000 neurons, the error is as small as one degree already at $t=10 \mathrm{~ms}$, an interval during which only a small proportion of selective neurons has emitted a spike. Note that one degree is the order of magnitude of the error made typically in perceptual discrimination tasks (see e.g. Pouget and Thorpe 1991). During the same interval the activity of the population of neurons carries about 6.5 bits about the stimulus. Doubling the number of neurons or the duration of the interval divides the minimal reconstruction error by $\sqrt{2}$ and increases the mutual information by 0.5 bit. 

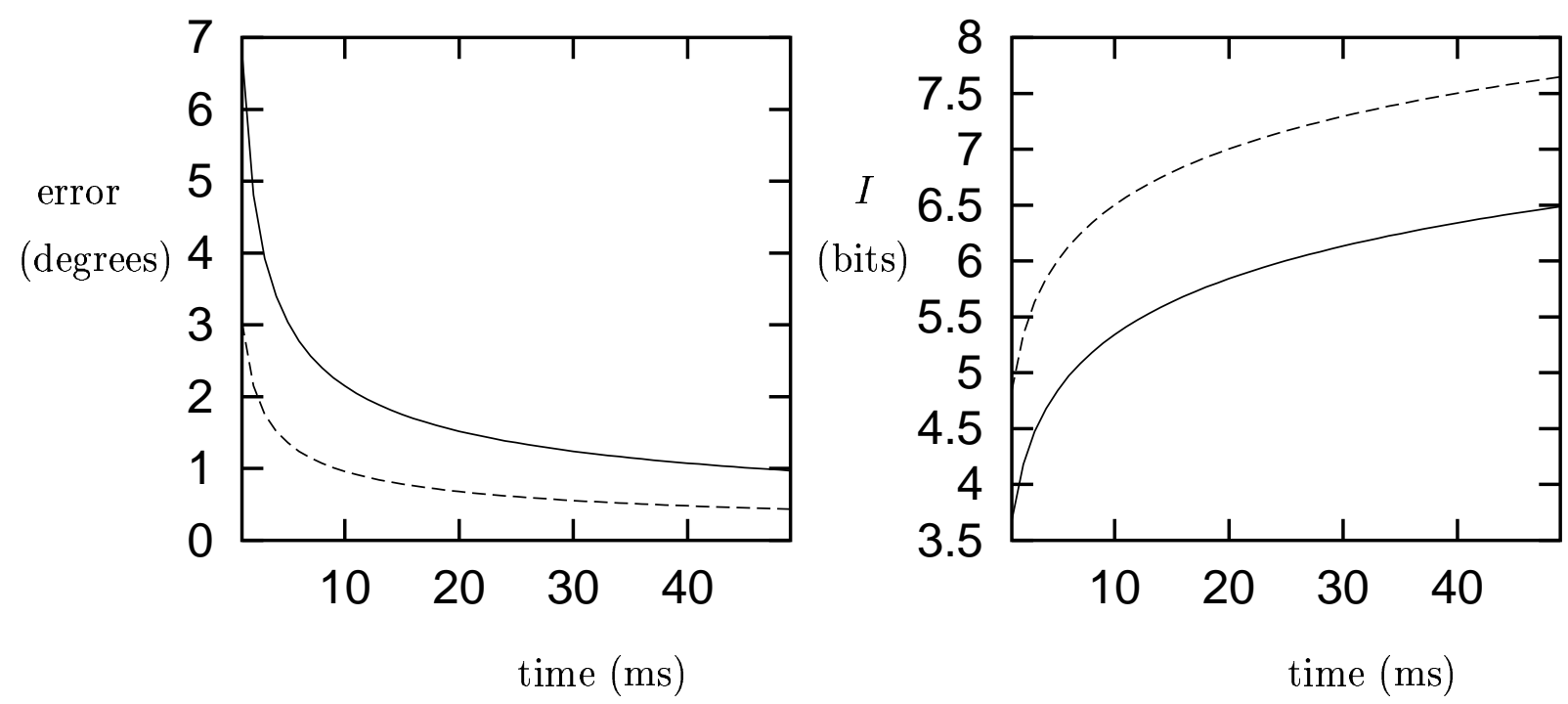

Figure 3: Left: minimal reconstruction error as given by the Cramer Rao bound for $N=1000$ (full curve), $N=5000$ (dashed curve) postsubiculum neurons, using data from (Taube et al 1990), as a function of time. Right: mutual information for $N=1000$ (full curve), $N=5000$ (dashed curve), using the same data and Eq. (13).

\section{Conclusion}

In this paper we have exhibited the link between Fisher information and mutual information in the context of neural coding. This link had been first derived in the context of Bayesian parameter estimation by Clarke and Barron (1990) and then in the context of stochastic complexity by Rissanen (1996). We have shown that the result of Rissanen applies to population coding, that is when the number of neurons is very large compared to the dimension of the stimulus. Our derivation of the link uses completely different techniques. The result is that the mutual information between the neural activities and the stimulus is equal to the one between the stimulus and an ideal Gaussian unbiased estimator whose variance is equal to the inverse of the Fisher information. The result is true not only for independent observations, but also for correlated activities (see Rissanen 1996 and the Appendix). This is important in the context of neural coding since noise in different cells might in some case be correlated, due to common inputs or to lateral connections.

This result implies that in the limit of a large number of neurons maximization of the mutual information leads to optimal performance in estimation of the stimulus. We have thus considered the problem of optimizing the tuning curves by maximizing 
the mutual information over the parameters defining the tuning curves: optimization of the choice of preferred orientations, widths of the tuning curves. In the simple model we have considered, the optimal value for the width is zero, as in (Seung and Sompolinsky 1993). However, we have shown that finite size effects necessarily lead to a non zero optimal value, independently of the decoding scheme.

We have discussed in detail the case of a one dimensional stimulus (an angle). A similar relationship between mutual information and the Fisher information matrix holds for any dimensionality of the stimulus, as long as it remains small compared to the number of neurons. It would be straightforward to consider in that more general case the optimization of the tuning curves. Zhang et al (1998) have computed the Fisher information matrix for 2 and 3 dimensional stimuli. Their results imply that optimal tuning curve parameters will depend strongly on the dimensionality of the stimulus.

We have shortly discussed the cases of a finite number of neurons and of the short time limit. In this case maximization of the mutual information leads in general to different results than minimization of the variance of reconstruction error, as found also in networks with the same number of input and output continuous neurons (Ruderman 1994). We are currently working on these limits for which many aspects remain to be clarified.

We have not addressed the problem of decoding. In the asymptotic limit, the maximum likelihood (ML) decoding is optimal. Recently Pouget and Zhang (1997) have shown that a simple recurrent network is able to perform the computation of the ML estimate. This suggests that the optimal performance, from the point of view of both information content and decoding, can be reached by a simple cortical architecture.

\section{Acknowledgements}

We thank Alexandre Pouget and Sophie Deneve for an interesting discussion, and Sid Wiener for drawing the data of (Taube et al 1990) to our attention. We are grateful to Alexandre Pouget and Peter Latham for pointing out a mistake in an earlier version of the mansucript, and to the referees for comments which helped us to improve significantly the paper. 


\section{References}

Atick JJ 1992, Could information theory provide an ecological theory of sensory processing? Network 3, 213-251

Barlow HB, Kaushal TP, and Mitchison GJ 1989, Finding minimum entropy codes. Neural Comp. 1, 412-423

Bhattacharya RN and Rao RR 1976, Normal approximation and asymptotic expansions, (Wiley)

Bialek W, Rieke F, de Ruyter van Steveninck R, and Warland D 1991, Reading a neural code. Science 252, 1854-57

Blahut RE 1988, Principles and Practice of Information Theory. Addison-Wesley, Cambridge MA

Clarke BS and Barron AR 1990, Information theoretic asymptotics of Bayes methods, IEEE Trans. on Information Theory 36, 453-471

Cover TM and Thomas JA 1991, Information Theory. John Wiley, New-York

Georgopoulos AP, Kalaska JF, Caminiti R and Massey JT 1982, On the relations between the direction of two-dimensional arm movements and cell discharge in primate motor cortex, J. Neurosci. 2, 1527-1537

Green DM and Swets JA 1966, Signal detection theory and psychophysics, John Wiley \& Sons, New-York.

Laughlin SB 1981, A simple coding procedure enhances a neuron's information capacity. Z. Naturf. C 36, 910-2

Linsker R 1988, Self-organization in a perceptual network. Computer 21, 105-17

Maunsell JHR and Van Essen DC 1983, Functional properties of neurons in middle temporal visual area of the macaque monkey. I. Selectivity for stimulus direction, speed, and orientation. J. Neurophysiol. 49, 1127-1147

Nadal J-P and Parga N 1994, Nonlinear neurons in the low noise limit: a factorial code maximizes information transfer Network 5, 565-581

Parisi G 1988, Statistical Field Theory, Addison-Wesley 
Pouget A and Thorpe SJ 1991, Connexionist models of orientation identification Connection Science 3, 127-142

Pouget A and Zhang K 1997, Statistically efficient estimations using cortical lateral connections NIPS 9, 97-103, Mozer MC, Jordan MI and Petsche T eds, MIT press

Rissanen J 1996, Fisher information and stochastic complexity, IEEE Trans. on Information Theory 42, 40-47

Ruderman D 1994, Designing receptive fields for highest fidelity. Network 5, 147155

Seung HS and Sompolinsky H 1993, Simple models for reading neural population codes. P.N.A.S. USA 90, 10749-10753

Shannon SE and Weaver W 1949, The Mathematical Theory of Communication, The University of Illinois Press, Urbana

Snippe HP 1996, Parameter extraction from population codes: a critical assesment. Neural Comp. 8, 511-529

Softky WR and Koch C 1993 The highly irregular firing of cortical cells is inconsistent with temporal integration of random EPSPs, J. Neurosci. 13, 334

Stein R 1967, The information capacity of nerve cells using a frequency code, Biophys. J. 7, 797-826

Taube JS 1995, Head direction cells recorded in the anterior thalamic nuclei of freely moving rats, J. Neurosci. 15, 70-86

Taube JS, Muller RU and Ranck JB 1990, Head direction cells recorded from the postsubiculum in freely moving rats. I. Description and quantitative analysis $J$. Neurosci. 10, 420-435

van Hateren JH 1992, Theoretical predictions of spatiotemporal receptive fields of fly LMCs, and experimental validation. J. Comp. Physiology A 171, 157-170

Zhang K, Ginzburg I, McNaughton BL and Sejnowski TJ 1998, Interpreting neuronal population activity by reconstruction: a unified framework with application to hippocampal place cells J. Neurophysiol. 79 1017-1044 


\section{Appendix}

Our goal is to derive (13), that is to compute the mutual information $I=I[P, \rho]$ between the random variables $\vec{x}$ and $\theta$, working in the large $N$ limit. We recall that $\vec{x}$ can be seen either as a set of $N$ observations related to the measurement of an unknown parameter $\theta$, or as the set of responses of $N$ neurons to a stimulus $\theta$. The mutual information $I$ is defined by

$$
I=\int d \theta \rho(\theta)\left\langle\ln \frac{P(\vec{x} \mid \theta)}{Q(\vec{x})}\right\rangle_{\theta}
$$

where $Q(\vec{x})$ is the pdf of $\vec{x}$ :

$$
Q(\vec{x})=\int d \theta \rho(\theta) P(\vec{x} \mid \theta) .
$$

In equation (39), $\langle\cdot\rangle_{\theta}$ denotes the integration over $\vec{x}$ given $\theta$ with the p.d.f. $P(\vec{x} \mid \theta)$. We define

$$
G(\vec{x} \mid \theta) \equiv \frac{1}{N} \ln P(\vec{x} \mid \theta)
$$

We will make the following hypothesis:

1. All derivatives of $G$ with respect to the stimulus $\theta$ are of order 1 in the large $N$ limit.

2. The cumulants of order $n$ of $x G_{\theta}^{\prime}+y G_{\theta}^{\prime \prime}$ are of order $1 / N^{n-1}$ in the large $N$ limit.

Both properties are verified for the factorized models (1) and (2), but also in some cases in which $x_{i}$ given $\theta$ are correlated variables, as we show at the end of the Appendix.

The large $N$ limit allows us to use the saddle-point method (Bhattacharya and Rao 1976, Parisi 1988) for the computation of integrals over $\theta$, in particular for the computation of the p.d.f. $Q(\vec{x})$, using the fact that $P(\vec{x} \mid \theta)$ will appear to be sharply peaked around its most probable value, the maximum-likelihood (ML) estimator of $\theta$. We will use standard cumulant expansions for the integration over $\vec{x}$ in the equivocation part of $I$, and this will eventually lead to the announced result, Eq. (13).

\section{Distribution of $\vec{x}$}

The p.d.f. $Q(\vec{x})$ can be written

$$
Q(\vec{x})=\int d \theta \rho(\theta) \exp N G(\vec{x} \mid \theta)
$$


For large $N$, the integral is dominated by the maxima of the integrand. These are defined by the solutions of

$$
G_{\theta}^{\prime}(\vec{x} \mid \theta)=0
$$

which satisfy $G_{\theta}^{\prime \prime}(\vec{x} \mid \theta)<0$. Above we have denoted by $G_{\theta}^{\prime}$ (resp. $G_{\theta}^{\prime \prime}$ ) the first (resp. second) partial derivative of $G$ with respect to $\theta$. Let us assume that $G(\vec{x} \mid \theta)$ has a single global maximum at $\theta_{m}(\vec{x})$ The Taylor expansion around $\theta_{m}(\vec{x})$ is

$$
G(\vec{x} \mid \theta)=G\left(\vec{x} \mid \theta_{m}(\vec{x})\right)+\frac{1}{2} G_{\theta}^{\prime \prime}\left(\vec{x} \mid \theta_{m}(\vec{x})\right)\left(\theta-\theta_{m}(\vec{x})\right)^{2}+\ldots
$$

Using standard saddle-point techniques we find

$$
Q(\vec{x})=Q_{m}(\vec{x})\left(1+O\left(\frac{1}{N}\right)\right)
$$

with

$$
Q_{m}(\vec{x}) \equiv \rho_{m}(\vec{x}) \sqrt{\frac{2 \pi}{N|\Gamma(\vec{x})|}} \exp \left[N G_{m}(\vec{x})\right]
$$

where

$$
\begin{aligned}
\rho_{m}(\vec{x}) & \equiv \rho\left(\theta_{m}(\vec{x})\right), \\
G_{m}(\vec{x}) & \equiv G\left(\vec{x} \mid \theta_{m}(\vec{x})\right)
\end{aligned}
$$

and

$$
\Gamma(\vec{x}) \equiv G_{\theta}^{\prime \prime}\left(\vec{x} \mid \theta_{m}(\vec{x})\right)
$$

Note that $\theta_{m}(\vec{x})$ is the maximum-likelihood (ML) estimator of $\theta$.

\section{The mutual information: integration over $\theta$}

Let us start with the following expression of the mutual information:

$$
I=-\int d \theta \rho(\theta) \ln \rho(\theta)+\int d^{N} x Q(\vec{x}) \int d \theta Q(\theta \mid \vec{x}) \ln Q(\theta \mid \vec{x})
$$

with $Q(\theta \mid \vec{x})=\frac{P(\vec{x} \mid \theta) \rho(\theta)}{Q(\vec{x})}$. The first term is the entropy of the input distribution. The second term can be written

$$
-\int d^{N} x Q(\vec{x}) \ln Q(\vec{x})+\int d^{N} x \int d \theta P(\vec{x} \mid \theta) \rho(\theta) \ln P(\vec{x} \mid \theta) \rho(\theta)
$$

In the above expression, the first part is the entropy of $\vec{x}$ in which we can replace $Q(\vec{x})$ by $Q_{m}(\vec{x})$ as given in (45), leading to

$$
-\int d^{N} x Q_{m}(\vec{x})\left[N G_{m}+\ln \rho_{m}-\frac{1}{2} \ln \frac{N|\Gamma(\vec{x})|}{2 \pi}\right]
$$


The last term in (49) can be written as

$$
\int d^{N} x \int d \theta A(\vec{x} \mid \theta) \exp A(\vec{x} \mid \theta)
$$

with

$$
A(\vec{x} \mid \theta) \equiv \ln P(\vec{x} \mid \theta) \rho(\theta)
$$

Now

$$
\int d \theta A(\vec{x} \mid \theta) \exp A(\vec{x} \mid \theta)=\left.\partial_{\lambda} \int d \theta \exp \lambda A\right|_{\lambda=1}
$$

which is again computed with the saddle point method,

$$
\begin{aligned}
\int d \theta A(\vec{x} \mid \theta) \exp A(\vec{x} \mid \theta) & =\left.\partial_{\lambda} \sqrt{\frac{2 \pi}{\lambda N|\Gamma(\vec{x})|}} \exp \lambda\left[N G_{m}+\ln \rho_{m}\right]\right|_{\lambda=1} \\
& =Q_{m}\left[N G_{m}+\ln \rho_{m}-\frac{1}{2}\right]
\end{aligned}
$$

Finally, putting everything together, the mutual information can be written

$$
I=-\int d \theta \rho(\theta) \ln \rho(\theta)+\int d^{N} x \rho\left(\theta_{m}(\vec{x})\right) \sqrt{\frac{2 \pi}{N|\Gamma(\vec{x})|}} \exp \left[N G_{m}(\vec{x})\right]\left(\frac{1}{2} \ln \frac{N|\Gamma(\vec{x})|}{2 \pi e}\right)
$$

It is already interesting to compare the above expression (50) with (13): as in (13) the first term above is the entropy $\mathcal{H}[\theta]=-\int d \theta \rho(\theta) \ln \rho(\theta)$ of the stimulus distribution; the second term, the equivocation, is in (50) given by the average over the $\vec{x}$ p.d.f. of the logarithm of the variance of the estimator for a given $\vec{x}$.

\section{The mutual information: integration over $\vec{x}$}

The last difficulty is now to perform in (50) the trace on $\vec{x}$. One cannot apply the saddle-point method directly because the number of variables on which integration is done is precisely equal to the number $N$ which makes the exponential large. However, the difficulty is circumvented by the introduction of a small (compared to $N$ ) auxilliary integration variables, in such a way that the integration over the $x_{i}$ 's can be done exactly. Then, we use again the fact that $N$ is large to perform the integration over the auxillary variables to leading order in $N$.

First we use the relation

$$
F\left(\theta_{m}(\vec{x})\right)=\int d \theta F(\theta)\left|G_{\theta}^{\prime \prime}(\vec{x} \mid \theta)\right| \delta\left(G_{\theta}^{\prime}(\vec{x} \mid \theta)\right)
$$


in order to deal with $\theta_{m}(\vec{x})$, which is valid for an arbitrary function $F$. We then use an integral representation of the delta function:

$$
\delta\left(G_{\theta}^{\prime}(\vec{x} \mid \theta)\right)=\int \frac{d y}{2 \pi} \exp \left(i y G_{\theta}^{\prime}(\vec{x} \mid \theta)\right)
$$

Similarly, in order to deal with $G_{\theta}^{\prime \prime}(\vec{x} \mid \theta)$ we introduce conjugate variables $\tau, \hat{\tau}$ : for any function $F$ we can write

$$
F\left(G_{\theta}^{\prime \prime}(\vec{x} \mid \theta)\right)=\int d \tau d \hat{\tau} \frac{1}{2 \pi} F(\tau) \exp \left(i \hat{\tau}\left(\tau-G_{\theta}^{\prime \prime}(\vec{x} \mid \theta)\right)\right) .
$$

Putting everything together we get

$$
I=\mathcal{H}[\theta]+\int d \theta d y d \tau d \hat{\tau} \frac{\sqrt{|\tau|}}{\sqrt{N}(2 \pi)^{\frac{3}{2}}} \rho(\theta)\left(\frac{1}{2} \ln \left(\frac{N|\tau|}{2 \pi e}\right)\right) \exp (i \hat{\tau} \tau+K(\theta, y, \hat{\tau}))
$$

in which

$$
K(\theta, y, \hat{\tau})=\ln \left\langle\exp \left(-i \hat{\tau} \frac{\partial^{2} G(\vec{x} \mid \theta)}{\partial \theta^{2}}+i y \frac{\partial G(\vec{x} \mid \theta)}{\partial \theta}\right)\right\rangle_{\theta}
$$

(we recall that $\langle\ldots\rangle_{\theta}=\int d^{N} x \exp [N G(\vec{x} \mid \theta)] \ldots$ ). We now make the cumulant expansion

$$
\langle\exp A\rangle_{\theta}=\exp \left(\langle A\rangle_{\theta}+\frac{1}{2}\left(\left\langle A^{2}\right\rangle_{\theta}-\langle A\rangle_{\theta}^{2}\right)+\ldots\right)
$$

for

$$
A \equiv-i \hat{\tau} G_{\theta}^{\prime \prime}+i y G_{\theta}^{\prime}
$$

The cumulant expansion will be valid if the cumulants of order $n$ of $A$ with the law $\exp [N G(\vec{x} \mid \theta)]$ decrease sufficiently rapidly with $n$. A sufficient condition is

assumption: the cumulants of order $n$ of $A(n=1,2, \ldots)$ are of order $1 / N^{n-1}$

Using the following identities obtained by deriving twice $1=\langle 1\rangle_{\theta}$ with respect to $\theta$,

$$
\begin{aligned}
& 0=\left\langle G_{\theta}^{\prime}\right\rangle_{\theta} \\
& 0=\left\langle G_{\theta}^{\prime \prime}\right\rangle_{\theta}+N\left\langle\left(G_{\theta}^{\prime}\right)^{2}\right\rangle_{\theta},
\end{aligned}
$$

one gets

$$
K=i \hat{\tau} J-\frac{\hat{\tau}^{2} \Delta^{2}}{2 N}-\frac{y^{2} J}{2 N}+\frac{y \hat{\tau} Z}{N}+O\left(\frac{1}{N^{2}}\right)
$$

where $J, \Delta, Z$ are given by

$$
\begin{aligned}
J & \equiv-\left\langle G_{\theta}^{\prime \prime}\right\rangle_{\theta}=N\left\langle\left(G_{\theta}^{\prime}\right)^{2}\right\rangle_{\theta} \\
\Delta^{2} & \equiv N\left(\left\langle\left(G_{\theta}^{\prime \prime}\right)^{2}\right\rangle_{\theta}-\left\langle G_{\theta}^{\prime \prime}\right\rangle_{\theta}^{2}\right) \\
Z & \equiv N\left\langle G_{\theta}^{\prime} G_{\theta}^{\prime \prime}\right\rangle_{\theta}
\end{aligned}
$$


Note that the Fisher information $\mathcal{J}(\theta)$ is equal to $N J$, and that $\Delta^{2}$ and $Z$ are of order 1 because of assumption (53).

In these terms we have

$$
\begin{aligned}
I=\mathcal{H}[\theta]+\int & d \theta d y d \tau d \hat{\tau} \frac{\sqrt{|\tau|}}{\sqrt{N}(2 \pi)^{\frac{3}{2}}} \rho(\theta)\left(\frac{1}{2} \ln \left(\frac{N|\tau|}{2 \pi e}\right)\right) \\
& \exp \left(i \hat{\tau}(\tau+J)-\frac{\hat{\tau}^{2} \Delta^{2}}{2 N}-\frac{y^{2} J}{2 N}+\frac{y \hat{\tau} Z}{N}+O\left(\frac{1}{N^{2}}\right)\right)
\end{aligned}
$$

Our last task is to integrate over the remaining auxilliary variables $\tau, \hat{\tau}, y$. Using the fact that $\Delta^{2}-\frac{Z^{2}}{J}>0$, deduced from the Schwartz inequality

$$
<G_{\theta}^{\prime}\left(G_{\theta}^{\prime \prime}-<G_{\theta}^{\prime \prime}>\right)>^{2} \leq<G_{\theta}^{2}><\left(G_{\theta}^{\prime \prime}-<G_{\theta}^{\prime \prime}>\right)^{2}>
$$

the integration over $y$ and $\hat{\tau}$ are simple Gaussian integrations, leading to:

$$
I=\mathcal{H}[\theta]+\int d \theta \rho(\theta) \int \frac{d \tau}{\sqrt{2 \pi}} \sqrt{\frac{N}{\Delta^{2}-\frac{Z^{2}}{J}}} \sqrt{\frac{|\tau|}{J}} \frac{1}{2} \ln \left(\frac{N|\tau|}{2 \pi e}\right) \exp \left(-\frac{N}{2} \frac{(\tau+J)^{2}}{\Delta^{2}-\frac{Z^{2}}{J}}\right)
$$

The integration over $\tau$ is with a Gaussian weight centered at $\tau=-J$ and with a width going to zero as $N$ goes to infinity:

$$
\lim _{N \rightarrow \infty} \frac{1}{\sqrt{2 \pi}} \sqrt{\frac{N}{\Delta^{2}-\frac{Z^{2}}{J}}} \exp -\frac{N}{2} \frac{(\tau+J)^{2}}{\Delta^{2}-\frac{Z^{2}}{J}}=\delta(\tau+J)
$$

Using the fact that the Fisher information is $\mathcal{J}(\theta)=N J$, we obtain

$$
I=-\int d \theta \rho(\theta) \ln \rho(\theta)-\int d \theta \rho(\theta) \frac{1}{2} \ln \left(\frac{2 \pi e}{\mathcal{J}(\theta)}\right)(1+O(1 / N))
$$

which is the announced result (13).

The conditions (53) of validity of the calculation are satisfied when $x_{i}$ given $\theta$ are independent, as in models (1) and (2), but can also be satisfied when they are correlated. We discuss below these two cases.

\section{Conditional independence of activities}

In the case of independent neurons, model (2), one can easily check that the cumulant expansion at order $n$ gives terms of order $1 / N^{n-1}$. Indeed, in that case one has

$$
G(\vec{x} \mid \theta)=\frac{1}{N} \sum_{i} g_{i}\left(x_{i} \mid \theta\right)
$$


so that

$$
A=\frac{1}{N} \sum_{i} A_{i}, \text { with } A_{i}=-i \hat{\tau} \frac{\partial^{2} g_{i}\left(x_{i} \mid \theta\right)}{\partial \theta^{2}}+i y \frac{\partial g_{i}\left(x_{i} \mid \theta\right)}{\partial \theta} .
$$

The cumulant expansion then reads

$$
\begin{aligned}
\langle\exp A\rangle & =\exp \sum_{i} \log \left\langle\exp \frac{A_{i}}{N}\right\rangle \\
& =\exp \sum_{i}\left(\frac{1}{N}\left\langle A_{i}\right\rangle+\frac{1}{N^{2}}\left(\left\langle A_{i}^{2}\right\rangle-\left\langle A_{i}\right\rangle^{2}\right)+O\left(1 / N^{3}\right)\right)
\end{aligned}
$$

Thus Eq. (54) holds, with $J, \Delta, Z$ given by

$$
\begin{aligned}
J & =-\frac{1}{N} \sum_{i}\left\langle\frac{\partial^{2} g_{i}}{\partial \theta^{2}}\right\rangle_{\theta}=\frac{1}{N} \sum_{i}\left\langle\left(\frac{\partial g_{i}}{\partial \theta}\right)^{2}\right\rangle_{\theta} \\
\Delta^{2} & =\frac{1}{N} \sum_{i}\left(\left\langle\left(\frac{\partial^{2} g_{i}}{\partial \theta^{2}}\right)^{2}\right\rangle_{\theta}-\left\langle\frac{\partial^{2} g_{i}}{\partial \theta^{2}}\right\rangle_{\theta}^{2}\right)^{2} \\
Z & =\frac{1}{N} \sum_{i}\left\langle\frac{\partial g_{i}}{\partial \theta} \frac{\partial^{2} g_{i}}{\partial \theta^{2}}\right\rangle_{\theta} .
\end{aligned}
$$

\section{Correlated neurons}

The conditions on the cumulants of $A$, Eq. (53), do not imply that the $x_{i}$ are independant, but they do have the qualitative meaning that they convey of order $N$ independent observations. To see this, we give here an example of correlated activities for which the conditons are satisfied.

We consider the following simple model. Each $x_{i}$ can be expressed in term of the same $N$ independant random variables, $\xi_{a}, a=1, \ldots, N$, as

$$
x_{i}=\sum_{a} M_{i, a} \xi_{a}
$$

where $M$ is a $\theta$-independent invertible matrix, and the $\xi$ 's are, given $\theta$, statistically independent variables of arbitrary p.d.f. $\rho_{a}(\xi \mid \theta), a=1, \ldots, N$. The factorized case is recovered for $M$ diagonal. In the case where the $\rho$ 's are Gaussian and $M$ is orthogonal, (60) is the principal component decomposition of the $x$ 's. We show now that the case $M$ invertible with arbitrary $\rho$ 's satisfies the conditions (53).

First, it is obvious that the result (13) holds: with the change of variables $\vec{x} \rightarrow$ $M^{-1} \vec{x}=\vec{\xi}$, one recovers the case of independent (given $\theta$ ) activities. One can then apply (13) to $I(\theta, \vec{\xi})$. Since $P(\vec{\xi} \mid \theta)=P(\vec{x} \mid \theta)|\operatorname{det} M|$, with $M$ independent of $\theta$, $I(\theta, \vec{x})=I(\theta, \vec{\xi})$ and the Fisher information associated to $P(\vec{\xi} \mid \theta)$ is equal to the one 
associated to $P(\vec{x} \mid \theta)$, so that (13) holds for $I(\theta, \vec{x})$. Second, one can check directly that conditions (53) holds. For our model, $G$ is

$$
G(\vec{x} \mid \theta)=-\frac{1}{N} \ln |\operatorname{det} M|+\frac{1}{N} \sum_{a} \ln \rho_{a}\left(\sum_{i} M_{a, i}^{-1} x_{i} \mid \theta\right)
$$

so that the cumulants of $\frac{\partial G(\vec{x} \mid \theta)}{\partial \theta}$ and $\frac{\partial^{2} G(\vec{x} \mid \theta)}{\partial \theta^{2}}$ with respect to the pdf $P(\vec{x} \mid \theta)$ are equal to the cumulants of $\frac{\partial G(\vec{\xi} \theta)}{\partial \theta}$ and $\frac{\partial^{2} G(\vec{\xi} \mid \theta)}{\partial \theta^{2}}$ with respect to the factorized pdf $P(\vec{\xi} \mid \theta)=$ $\prod_{a} \rho_{a}(\xi \mid \theta)$ for which (53) holds. 